\title{
Clinical investigation and imaging
}

\author{
Daniel Studer
}

Received: 1 April 2012/Accepted: 17 May 2012/Published online: 11 December 2012

(C) EPOS 2012

\begin{abstract}
Adolescent idiopathic scoliosis (AIS) affects $2-4 \%$ of children and is diagnosed between age 10 and skeletal maturity. The female to male ratio for mild curves less than $20^{\circ}$ is $1.5: 1$; however, progression to a severe deformity occurs more often in females (Weinstein in JAMA 289(5):559-567, 2003). Despite significant ongoing research, including into the genetic basis for AIS, there are currently no identifiable causes, and therefore the disorder still remains a diagnosis of exclusion. History, physical examination and radiographic assessment must exclude other possible causes of spinal deformity and are crucial in predicting the risk of curve progression. History should focus on family history, menarche, presence or absence of pain, sports activities and neurologic changes. Physical examination concentrates on anthropometric data, pubertal staging, neurologic testing and specific investigation of the spine, with the Adams' forward bending test being the most meaningful step to evaluate trunk rotation. Definitive diagnosis cannot be made without imaging. The gold standard remains plain radiography with assessment of the Cobb angle on a standing coronal radiograph of the entire spine. A lateral X-ray is used for assessing sagittal balance and for evaluating the deformity in the sagittal plane. If available, surface topography can accompany the followup in AIS, reducing the radiation exposure. The role of magnetic resonance imaging (MRI) in AIS is an ongoing matter of debate. Common indications for MRI are the presence of an atypical curve pattern and abnormal neurological findings.
\end{abstract}

D. Studer $(\bowtie)$

Orthopaedic Department, University Children's Hospital,

4031 Basel, Switzerland

e-mail: daniel.studer@ukbb.ch
Keywords Adolescent idiopathic scoliosis - Patient's history · Clinical investigation · Imaging

\section{Clinical investigation}

Medical history

Evaluation of a child with scoliosis should begin with a comprehensive and complete medical history focused on family history of scoliosis, complaints of pain, neurologic signs or symptoms, including bowel and bladder dysfunction, information on physical development and sports activities.

Family history

AIS is often seen in multiple members of one family, strongly suggesting a genetic component. A meta-analysis of studies of twins showed concordance for AIS in $73 \%$ of monozygotic and $36 \%$ of dizygotic twins [2]. A recent study confirmed the genetic etiology in AIS, but with a concordance being much lower [3]. Current research is focusing on identifying the multiple responsible genes that cause AIS and that probably play an important role in determining the risk of curve progression. In 2010 a DNAbased test (ScoliScore, Axial Biotech, Salt Lake City, USA) to predict spinal curve progression in AIS was presented and validated [4]. This promising tool will probably revolutionize future concepts of AIS treatment but so far it is applicable only to Caucasian females and males between ages 9-13 which have already been diagnosed with mild scoliosis (10-25 Cobb angle). 
Pain

Pain is not typically associated with idiopathic scoliosis. If pain is present, its type and character are important in order to initialize further investigation. Muscular pain can usually be distinguished from neurologic pain by its location, mostly along the paravertebral muscles, and the relation to activity. Neurologic pain is more consistent and often associated with weakness and/or paraesthesia.

\section{Neurologic sings/symptoms}

As with pain there are normally no specific neurologic findings in AIS. But inquiry on bowel and bladder (dys-) function complement the neurologic history.

\section{Physical development}

The risk of curve progression in AIS is primarily correlated to factors predicting remaining skeletal growth potential. In girls, the menstrual onset remains the most important, and in most cases remembered, parameter, whereas the puberty vocal change in boys is often misdated.

\section{Sports activities}

Due to the fact that AIS is attributed to relative anterior spinal overgrowth [5], sports activities with repetitive hyperextension of the spine can assist in the development of AIS. Tanchev et al. [6] reported a tenfold higher incidence for idiopathic scoliosis in rhythmic gymnastic trainees than in their normal coevals. Even though still obscure, the generally noted joint laxity in gymnasts may be seen as a co-factor in the development or progression of AIS [7].

\section{Physical examination}

In combination with the medical history, the physical examination is essential for addressing the three-dimensional nature of AIS and evaluating the remaining growth potential. The characteristic changes in rhythm of growth, including phases of acceleration and deceleration, well described by Dimeglio et al. [8], must be considered. They described puberty to be the turning point during which the curvature will be emphasized and pointed up the importance of identifying its first signs to anticipate the curvatures that are going to progress.

Physical examination should therefore start with documenting body weight and height. An increase in the growth rate of the standing height greater than $0.5 \mathrm{~cm}$ per month or $6 \mathrm{~cm}$ per year is the first sign of puberty [8]. Measuring the sitting height also helps in further distinguishing the growth rate of the spine compared to the absolute growth. The Tanner stages define the physical development based on external primary and secondary sex characteristics $[9$, $10]$.

Before performing specific tests to objectify the spinal deformity a neurologic examination is mandatory. To assess overall balance and gross motor strength, an evaluation of gait patterns including toe- and heel-walking and bi- and monopedal hopping as well as a thorough motor and sensory examination are important. Deep tendon reflexes should also be assessed and compared with the contralateral side. Missing or abnormal abdominal reflexes are seen in $11 \%$ of patients with AIS. However, in $25-83 \%$ of patients with presumed AIS intraspinal disorders, especially syringomyelia, were detected on MRI when abdominal reflexes were absent or abnormal [11, 12]. Additionally, clinical findings like cavus foot deformity, hairy patches over the lower back, neurofibromas and asymmetric muscular atrophy can be signs of associated intraspinal disorders [13].

The examination of the spine is best performed when sitting on a chair facing the patient's back. To rule out a functional scoliosis the iliac crests should be levelled in case of leg-length discrepancy. The patient's back is addressed for shoulder obliquity, measured in centimetres, and trunk asymmetry (flattening of the waist on the convexe side; accentuation on the concave side). Coronal decompensation, reflected by a shift of the trunk to either side, is measured with a plumb line from the spinous process of $\mathrm{C} 7$ with deviation to the rima ani, quoted in centimetres. Trunk rotation-the most important clinical value in scoliosis-is best addressed with Adams' forward bending test, first described by Adams [14]. For many years the height of the rib hump at the apex of the scoliosis was used to describe the deformity. This cumbersome technique failed in accuracy, as did most of the subsequent methods, and it took almost 120 years until William Bunnell presented a device, easy to use and with a high reproducibility, that found its way into the coat pocket of probably every spine surgeon-the scoliometer [15]. Beside the possibility of measuring the thoracic deformity, the lumbar aspect of trunk rotation could also now be objectified. Different studies reconfirmed that the accuracy and reliability of the scoliometer was adequate $[16,17]$. With this key tool, a standard deviation of $1.9^{\circ}$ for the thoracic spine and $2.3^{\circ}$ for the lumbar spine have been reported [16].

Motivated by his successful invention, Bunnell even correlated the amount of trunk rotation measured with the scoliometer to the Cobb angle seen on plain radiographs, describing a correlation coefficient of 0.89 . The mean Cobb angle in patients with a $5^{\circ}$ angle of trunk rotation was $11^{\circ}$, whereas the mean trunk rotation in patients with a $20^{\circ}$ Cobb angle was $7^{\circ}[15]$. As a rule of thumb for estimating 

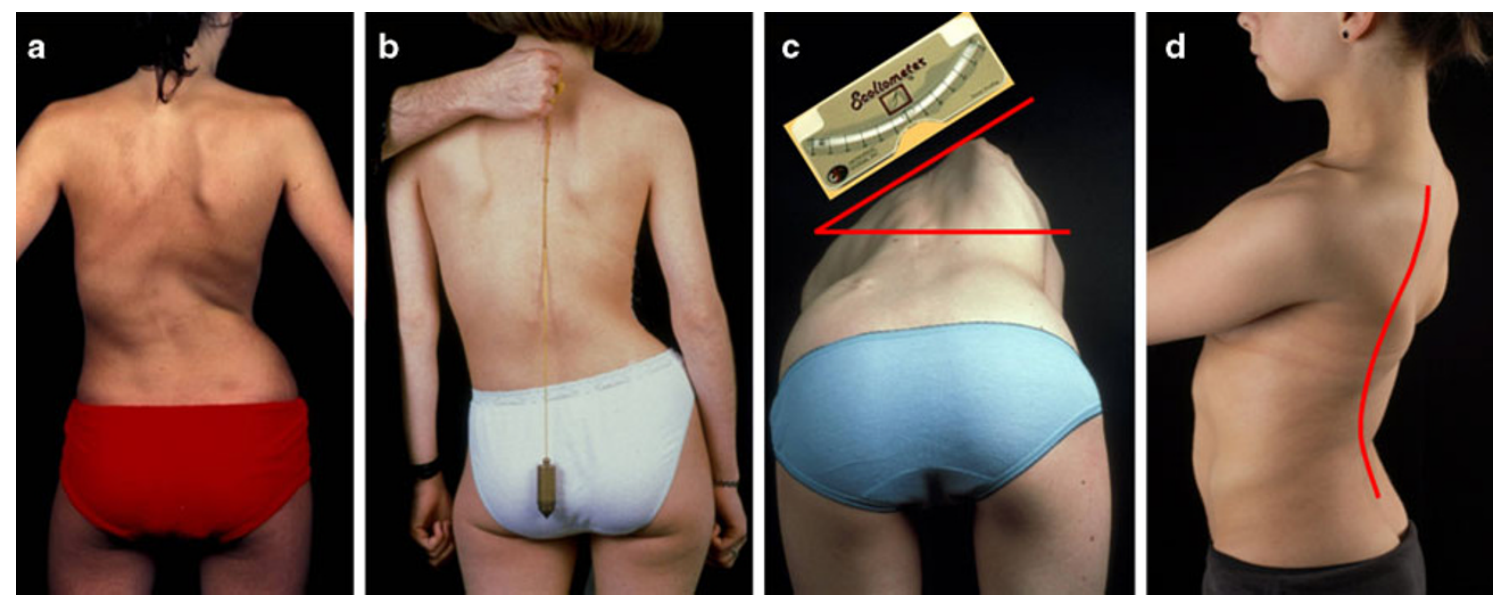

Fig. 1 Clinical investigation of the spinal deformity showing trunk asymmetry (a), coronal imbalance (b), rib hump as clinical sign of apical segmental rotation, measured with the scoliometer while forward bending (c), and relative thoracic lordosis (d)

the Cobb angle, the values measured with the scoliometer can be multiplied by 3 for the thoracic spine and by 2 for the lumbar spine. The large individual spread must, however, be respected [18].

In "Conclusion", we usually indicate an X-ray when measuring an angle of trunk rotation $\geq 5^{\circ}$.

Clinical investigation of the spine ends with assessing the sagittal profile in regard of sagittal (im-) balance as well as sagittal alignment. In case of sagittal imbalance a plumb line from the center of the shoulder falls either ventral or dorsal to the hip joint. Sagittal alignment expresses the aspect of the lateral curvatures of the spine. Thoracic AIS, especially, often presents with a relative lordosis of the thoracic spine due to rotation of the affected segments (Fig. 1).

\section{Imaging}

Detection of scoliosis remains a clinical feature but confirmation of the diagnosis as well as subsequent monitoring cannot be made without imaging. The role of different available modalities in the management of AIS will be highlighted.

\section{Plain radiography}

Plain radiography still represents the gold standard in confirming the diagnosis in idiopathic scoliosis. Radiographic assessment of AIS should involve full-length standing - to address the effects of gravity-anteroposterior (ap) and lateral views (3-foot-long cassette) of the entire spine including the pelvis and the hip joints. Respecting the great variety of radiographic information, the main characteristics to be analyzed on the ap-view are the curve pattern and angle with its respective apex, as well as coronal balance, overall rotation and Risser's sign/triradiate cartilage.
The typical curve pattern in AIS is usually a right convex thoracic curve with or without an additional left convex lumbar curve. Amongst the various techniques of assessing the magnitude of the curvature, the most widely used method has been described by J. R. Cobb in 1948. Therefore, identification of the end vertebrae (most tilted vertebrae at the cephalad and caudad end of the curve) is mandatory. The Cobb angle is defined by perpendiculars to lines drawn from the superior endplate of the cephalad end vertebra and from the inferior endplate of the caudad end vertebra. If endplates are difficult to visualize, the borders of the pedicles may be used. In addition to the end vertebra, also the stable vertebra (the first vertebra caudad to the curve that is bisected by the central sacral vertical line) as well as the neutral vertebra (the vertebra that shows no rotation) should be objectified, especially in case of preoperative planning (Fig. 2).

When comparing two radiographs, a $5^{\circ}$ difference in the Cobb angle represents a $95 \%$ chance that there is a true difference [19] and, vice versa, if there is less than $5^{\circ}$ difference, no real change is present. In AIS, the primary clinical utility of the Cobb angle is determination of the risk of curve progression. The Cobb angle otherwise has limited prognostic value. It does not correlate with the degree of morbidity or pain [20].

Considering the size of the curvature, a main curve as well as secondary curves are specified. According to the Scoliosis Research Society (SRS), the curve itself is defined by the level of its respective apex:

$\begin{array}{ll}\text { Thoracic } & \text { T2 }- \text { Disc T11/12 } \\ \text { Thoracolumbar } & \text { T12 - L1 } \\ \text { Lumbar Disc } & \text { L1/2 - L4 }\end{array}$

Coronal balance describes the alignment of the $\mathrm{C} 7$ plumb line with the central sacral vertical line (CSVL). In case of coronal compensation these two lines overlap, 
Fig. 2 a Cobb angle measurement: Lines are drawn extending the endplate of the superior and inferior end vertebra. Perpendiculars to these lines define the Cobb angle. b The end vertebra [EV] is the most tilted vertebra, the stable vertebra $[\mathrm{SV}]$ is bisected by the central sacral vertical line [CSVL], and the neutral vertebra $[\mathrm{NV}]$ shows no rotation (symmetric pedicles)
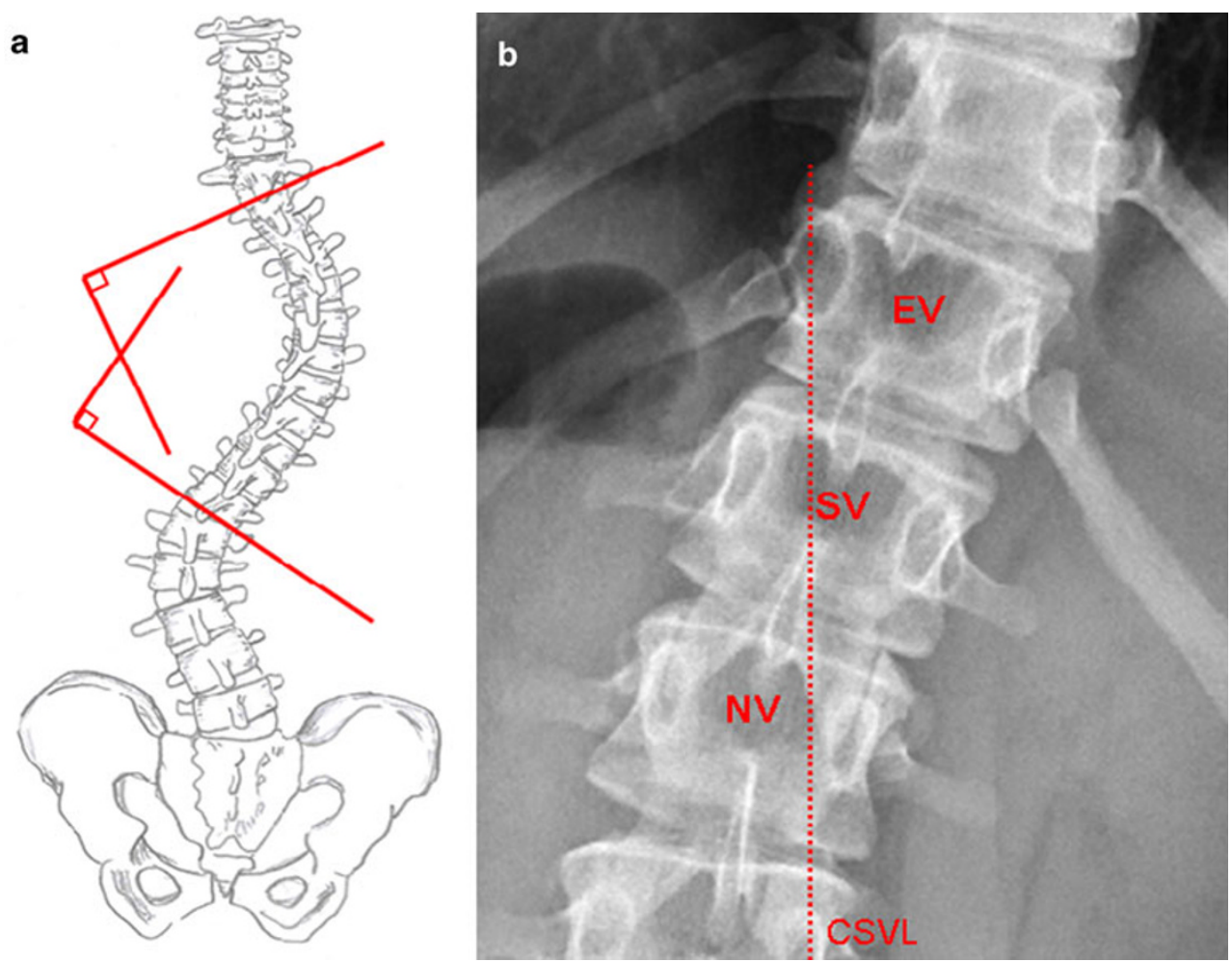

whereas in coronal decompensation a shift either to the left (negative) or to the right (positive) exists. Perdriolle and Nash-Moe techniques remain the standard measurements for providing a reasonable estimate of vertebral rotation $[21,22]$.

Gradually, ossification of the iliac crest (Risser's sign), as well as the condition of the triradiate cartilage, help in estimating residual growth potential. Risser noted that the growth plates of the spine were not easily visible and could not be used to reliably assess spinal growth. However, the completion of the excursion of the ossification of the iliac apophysis generally was coincident with that of the vertebral growth plates. With contact between the apophyseal ossification and the ilium, Risser considered that one could presume vertebral growth to be complete [23]. The disadvantage of the Risser classification is that the whole period of vertebral growth acceleration, with the highest risk of curve progression, happens before the ossification of the iliac crest starts (Risser 0). The triradiate cartilage closes before the appearance of the iliac ossification center during the before mentioned period of accelerated spinal growth [24]. In order to further divide this high risk phase of curve progression, addressing the ossification of the olecranon, as described by Sauvegrain [25], has become more and more popular and should be integrated in the decision-making process in the treatment of AIS [8].

The lateral X-ray of the spine is used for evaluating the deformity in the sagittal plane and for assessing sagittal balance. Normal thoracic kyphosis is $20-50^{\circ}$, measured from the upper endplate of $\mathrm{T} 2$ to the lower endplate of $\mathrm{T} 12$ using the Cobb method. Lumbar sagittal alignment is measured from the upper endplate of T12 and the upper endplate of S1, with a normal range being approximately $40-60^{\circ}$. In neutral sagittal balance a vertical line from the center of $\mathrm{C} 7$ passes through the posterior-superior corner of $\mathrm{S} 1$. Plumb lines falling either posterior or anterior are related to negative or positive sagittal balance, respectively. Sagittal malalignment presents as an exaggeration or deficiency of normal lordosis or kyphosis. The resultant pelvic and lower limb posture is an attempt to restore normal alignment [26]. Recently, the influences on the sagittal balance due to the relation between the spine and the pelvis have been better evaluated. Among the many investigated parameters, pelvic incidence, defined as the angle between the perpendicular of the upper sacral plate and the line joining the middle of the upper sacral plate and the hip axis, is of prime importance in determining the orientation of the pelvis (pelvic tilt $=$ angle between a vertical line and the line joining the middle of the upper sacral plate and the hip axis) and of the sacrum (sacral slope $=$ angle between the sacral plate and a horizontal line and therefore correlated with the shape and orientation of the lumbar spine [27].

In order to classify curves and, even more important, to select fusion levels in progressive AIS, radiographic assessment of spinal flexibility is mandatory. Various 
techniques have been developed and were evaluated and compared by several studies [28-30]. Liu and colleagues summarise in their review article that supine side-bending radiographs have been considered the major method for determining curve flexibility. However, when compared with postoperative correction using modern segmental spinal instrumentation techniques, supine side-bending flexibility radiographs have been less accurate in predicting correction. Push-prone radiographs were originally described by Kleinman et al. [31]. The benefit of showing all curves on a single film was impaired by the lower correction rates [32]. Fulcrum-bending radiographs, as described by Cheung and Luk [33], demonstrated statistically better correction of the main thoracic curves [30]. Klebbs et al. [32] concluded that fulcrum-bending radiographs should be obtained for main thoracic curves, whereas standing side-bending views should be used for evaluating thoracolumbar/lumbar curves. Newer studies report on even better results with suspension techniques or traction views under general anaesthesia [29, 34]. The concerns with suspension views are the so far unknown forces applied to the spine as well as patient's discomfort. Traction under general anesthesia not only provides equivalent information on curve correction but also allows better estimate of spinal balance [28]. The obvious negative fact is the missing preoperative availability.

Restrictively, all information gained in these studies deal with overall flexibility of the spine. Only recently, the lack of data on the segmental response of scoliotic curves to load has been addressed. Beside a curve flexibility index [(upright Cobb angle-bending Cobb angle $) \times 100 /$ upright Cobb angle] the authors also described a segmental flexibility index [(upright disc angle-bending disc angle) $\times 100 /$ upright disc angle], showing little flexibility in the four periapical scoliotic segments with more mobile segments towards the end of the curve [35].

Follow-up of AIS entails repetitive X-ray exposure. This has become a source of concern, with several studies showing that harmful effects can be linked to ionizing radiation, especially for younger patients [36, 37]. Since dose-free imaging methods like magnetic resonance imaging and ultrasound not only underestimate the curve size because of missing gravity, but also fall short in producing high-resolution images of bony structures, the practical solution resides in minimizing the dose while preserving or even enhancing image quality. A first big step has been realized by using digital X-ray techniques with a decrease in radiation exposure of approximately one-third compared with conventional plain radiography [38]. A recent study with a slot-scanning X-ray imager (EOS, Biospace, Paris, France) not only depicted another reduction in average skin dose of as high as 6-9 times in the thoracoabdominal region, but also showed an improvement in image quality compared with computed radiography [39]. Additionally, the EOS system permits a simultaneous acquisition of upright frontal and sagittal views, being able to cover in the same time the spine and the lower limbs and study the axial plane on 3D envelope reconstructions.

\section{Surface topography}

We have learned that curves in AIS are described by their appearance on plain films and quantified by the magnitude of the Cobb angle. This approach implicates the need for repeated radiographic examination. The use of surface topography can reduce the number of radiographs required in the monitoring of patients with AIS and so reduce radiation exposure.

Many devices have been used and can be considered in two broad groups: those that require direct measurement of the patient's back, and those utilising reconstruction of surface shape from scanned light or photographic techniques. A precursor of modern techniques was the moiré topography that was widely used for school screening programs all over the world in the 1970s and 1980s of the last century [40-43]. Among the various subsequently available techniques, video raster stereography is probably the most widely used and evaluated method. It provides a three-dimensional analysis of the shape of the trunk, allowing a reconstruction of the spinal deformity. The accuracy of the method has been confirmed in patients with idiopathic scoliosis of up to $80^{\circ}$ [44-46]. The apical vertebral lateral deviation measured with stereoradiography showed high correlation to the apical axial rotation on plain radiographs [47, 48]. Nevertheless, as shown by Goldberg et al. [49] only part of the deformity documented with surface topography can directly be expressed by the radiographically measured Cobb angle. The authors therefore abstained from deducing an algorithm to convert topographic to radiographic measures and preferred to consider each child individually as her or his own control during follow-up.

Another limiting factor in the use of surface topography might be the high costs of the device resulting in additional financial investment many hospitals can not afford, whereas an X-ray apparatus is an inherent part of almost every clinic.

\section{Magnetic resonance imaging (MRI)}

The prevalence of central nervous system (CNS) abnormalities in patients with presumed AIS is between 2 and $4 \%$ [50-52]. Therefore, the positive rate for screening all patients is low; however, the risk associated with missing one of these neurologic diagnose and proceeding with surgery may be significant. Hence, at our institution we routinely perform a preoperative MRI even though substantial 
controversy exists regarding the indications for imaging of the CNS in patients with AIS [50]. In 2004 Davids et al. [52] presented a study including 1280 patients with presumed AIS. MRI was ordered in 271 patients on the basis of the presence of selected indicators determined from the clinical history, physical examination, and plain radiographic assessment of the spine. Abnormal findings were seen in $10 \%$ of these patients, representing $2 \%$ of the total cohort. Interestingly, none of the children in whom pain was the only indicator category had an abnormal finding on MRI. In the study, $10 \%$ of patients with an atypical curve pattern (left thoracic, short segmented, absence of thoracic apical segment lordosis) and $6 \%$ of those with an isolated neurological finding (abnormal abdominal reflexes, hyperreflexia, asymmetric deep tendon reflexes, paresthesias, cavus foot deformity, urinary dysfunction, diminished rectal tone, skin lesions over the lower back) showed abnormalities on MRI. The most valuable single indicator of an abnormal finding was absence of thoracic apical segment lordosis (21\%). The optimal diagnostic yield occurred when both an atypical curve pattern and neurological indicators were present (25\%).

\section{Conclusions}

With the help of Adams' forward bending test, the detection of trunk rotation as a strong indicator for underlying scoliosis can be achieved within a very short time and should therefore be part of any routine paediatric examination.

Based upon the interplay of clinical investigation, including detailed enquiry of medical history and extensive physical examination, and imaging, secondary causes must be ruled out in order to identify idiopathic scoliosis. Plain radiography remains the gold standard when evaluating and classifying AIS. Surface topography can be implemented during follow-up, reducing radiation exposure. MRI should be performed preoperatively and in case of either combined or isolated atypical curve pattern and abnormal neurological findings.

The ongoing research in identifying the aetiology of AIS, especially in the field of genetics, will presumably interfere with today's concepts, not only when dealing with detection of scoliosis, but also in respect of future treatment plans.

Conflict of interest I have not received funds for this study.

\section{References}

1. Weinstein SL et al (2003) Health and function of patients with untreated idiopathic scoliosis: a 50-year natural history study. JAMA 289(5):559-567
2. Kesling KL (1997) A meta-analysis of the literature and report of six cases. Spine (Phila Pa 1976) 22(17):2009-2014 (discussion 2015)

3. Andersen MO, Thomsen K, Kyvik KO (2007) Adolescent idiopathic scoliosis in twins: a population-based survey. Spine (Phila Pa 1976) 32(8):927-930

4. Ward K et al (2010) Validation of DNA-based prognostic testing to predict spinal curve progression in adolescent idiopathic scoliosis. Spine (Phila Pa 1976) 35(25):E1455-E1464

5. Guo X et al (2005) Relative anterior spinal overgrowth in adolescent idiopathic scoliosis-result of disproportionate endochondral-membranous bone growth? Summary of an electronic focus group debate of the IBSE. Eur Spine J 14(9):862-873

6. Tanchev PI et al (2000) Scoliosis in rhythmic gymnasts. Spine (Phila Pa 1976) 25(11):1367-1372

7. Meyer C et al (2006) Why do idiopathic scoliosis patients participate more in gymnastics? Scand J Med Sci Sports 16(4):231-236

8. DiMeglio A, Canavese F, Charles YP (2011) Growth and adolescent idiopathic scoliosis: when and how much? J Pediatr Orthop 31(1 Suppl): S28-36

9. Marshall WA, Tanner JM (1969) Variations in pattern of pubertal changes in girls. Arch Dis Child 44(235):291-303

10. Marshall WA, Tanner JM (1970) Variations in the pattern of pubertal changes in boys. Arch Dis Child 45(239):13-23

11. Saifuddin A et al (2005) Prevalence and clinical significance of superficial abdominal reflex abnormalities in idiopathic scoliosis. Eur Spine J 14(9):849-853

12. Zadeh HG et al (1995) Absent superficial abdominal reflexes in children with scoliosis. An early indicator of syringomyelia. J Bone Joint Surg Br 77(5):762-767

13. Fribourg D, Delgado E (2004) Occult spinal cord abnormalities in children referred for orthopedic complaints. Am J Orthop (Belle Mead NJ) 33(1):18-25

14. Adams W (1865) Lectures on the pathology and treatment of lateral and other forms of curvature of the spine. Churchill, London

15. Bunnell WP (1984) An objective criterion for scoliosis screening. J Bone Joint Surg Am 66(9):1381-1387

16. Murrell GA et al (1993) An assessment of the reliability of the Scoliometer. Spine (Phila Pa 1976) 18(6):709-712

17. Cote P et al (1998) A study of the diagnostic accuracy and reliability of the Scoliometer and Adam's forward bend test. Spine (Phila Pa 1976) 23(7):796-802 discussion 803

18. Sapkas G et al (2003) Prediction of Cobb angle in idiopathic adolescent scoliosis. Clin Orthop Relat Res 411:32-39

19. Pruijs JE et al (1994) Variation in Cobb angle measurements in scoliosis. Skeletal Radiol 23(7):517-520

20. Malfair D et al (2010) Radiographic evaluation of scoliosis: review. AJR Am J Roentgenol 194(3 Suppl):S8-S22

21. Nash CL Jr, Moe JH (1969) A study of vertebral rotation. J Bone Joint Surg Am 51(2):223-229

22. Perdriolle R, Vidal J (1987) Morphology of scoliosis: threedimensional evolution. Orthopedics 10(6):909-915

23. Risser JC (1958) The Iliac apophysis; an invaluable sign in the management of scoliosis. Clin Orthop 11:111-119

24. Vital JM et al (1989) Radiologic change of cartilage of the pelvis and the upper end of the femur during growth. Application to the surveillance of scolioses. Chir Pediatr 30(2):103-108

25. Sauvegrain J, Nahum H, Bronstein H (1962) Study of bone maturation of the elbow. Ann Radiol (Paris) 5:542-550

26. Roussouly P, Nnadi C (2010) Sagittal plane deformity: an overview of interpretation and management. Eur Spine $J$ 19(11):1824-1836

27. Mac-Thiong JM et al (2007) Sagittal spinopelvic balance in normal children and adolescents. Eur Spine J 16(2):227-234 
28. Liu RW et al (2010) Comparison of supine bending, push-prone, and traction under general anesthesia radiographs in predicting curve flexibility and postoperative correction in adolescent idiopathic scoliosis. Spine (Phila Pa 1976) 35(4):416-422

29. Lamarre ME et al (2009) Assessment of spinal flexibility in adolescent idiopathic scoliosis: suspension versus side-bending radiography. Spine (Phila $\mathrm{Pa}$ 1976) 34(6):591-597

30. Li J et al (2011) Coronal deformity correction in adolescent idiopathic scoliosis patients using the fulcrum-bending radiograph: a prospective comparative analysis of the proximal thoracic, main thoracic, and thoracolumbar/lumbar curves. Eur Spine J 20(1):105-111

31. Kleinman RG et al (1982) The radiographic assessment of spinal flexibility in scoliosis: a study of the efficacy of the prone push film. Clin Orthop Relat Res 162:47-53

32. Klepps SJ et al (2001) Prospective comparison of flexibility radiographs in adolescent idiopathic scoliosis. Spine (Phila $\mathrm{Pa}$ 1976) 26(5):E74-E79

33. Cheung KM, Luk KD (1997) Prediction of correction of scoliosis with use of the fulcrum bending radiograph. J Bone Joint Surg Am 79(8):1144-1150

34. Davis BJ et al (2004) Traction radiography performed under general anesthetic: a new technique for assessing idiopathic scoliosis curves. Spine (Phila Pa 1976) 29(21):2466-2470

35. Hasler CC, Hefti F, Buchler P (2010) Coronal plane segmental flexibility in thoracic adolescent idiopathic scoliosis assessed by fulcrum-bending radiographs. Eur Spine J 19(5):732-738

36. Bone CM, Hsieh GH (2000) The risk of carcinogenesis from radiographs to pediatric orthopaedic patients. J Pediatr Orthop 20(2):251-254

37. Hoffman DA et al (1989) Breast cancer in women with scoliosis exposed to multiple diagnostic $\mathrm{X}$ rays. $\mathrm{J}$ Natl Cancer Inst 81(17):1307-1312

38. Kluba $\mathrm{T}$ et al (2006) Prospective randomized comparison of radiation exposure from full spine radiographs obtained in three different techniques. Eur Spine J 15(6):752-756

39. Deschenes S et al (2010) Diagnostic imaging of spinal deformities: reducing patients radiation dose with a new slot-scanning X-ray imager. Spine (Phila Pa 1976) 35(9):989-994
40. Willner S (1979) Moire topography for the diagnosis and documentation of scoliosis. Acta Orthop Scand 50(3):295-302

41. Willner S (1979) Moire topography: a method for school screening of scoliosis. Arch Orthop Trauma Surg 95(3):181-185

42. Daruwalla JS, Balasubramaniam P (1985) Moire topography in scoliosis. Its accuracy in detecting the site and size of the curve. J Bone Joint Surg Br 67(2):211-213

43. Adair IV, Van Wijk MC, Armstrong GW (1977) Moire topography in scoliosis screening. Clin Orthop Relat Res 129:165-171

44. Hackenberg L et al (2003) Rasterstereographic back shape analysis in idiopathic scoliosis after posterior correction and fusion. Clin Biomech (Bristol, Avon) 18(10):883-889

45. Hackenberg L et al. (2003) Rasterstereographic back shape analysis in idiopathic scoliosis after anterior correction and fusion. Clin Biomech (Bristol, Avon) 18(1):1-8

46. Schulte TL et al (2008) Raster stereography versus radiography in the long-term follow-up of idiopathic scoliosis. J Spinal Disord Tech 21(1):23-28

47. Stokes IA (1989) Axial rotation component of thoracic scoliosis. J Orthop Res 7(5):702-708

48. Stokes IA, Armstrong JG, Moreland MS (1988) Spinal deformity and back surface asymmetry in idiopathic scoliosis. J Orthop Res 6(1):129-137

49. Goldberg CJ et al (2001) Surface topography, Cobb angles, and cosmetic change in scoliosis. Spine (Phila Pa 1976) 26(4):E55E63

50. Do $\mathrm{T}$ et al (2001) Clinical value of routine preoperative magnetic resonance imaging in adolescent idiopathic scoliosis. A prospective study of three hundred and twenty-seven patients. J Bone Joint Surg Am 83-A(4):577-579

51. Maiocco B et al (1997) Adolescent idiopathic scoliosis and the presence of spinal cord abnormalities. Preoperative magnetic resonance imaging analysis. Spine (Phila Pa 1976) 22(21):25372541

52. Davids JR, Chamberlin E, Blackhurst DW (2004) Indications for magnetic resonance imaging in presumed adolescent idiopathic scoliosis. J Bone Joint Surg Am 86-A(10):2187-2195 